\title{
On Wave Propagation in Repetitive Structures: Two Forms of Transfer Matrix
}

\author{
A.K. Ashari and N.G. Stephen* \\ Mechanical Engineering, Faculty of Engineering and Physical Sciences, \\ University of Southampton, Highfield, Southampton, SO17 1BJ, UK \\ and \\ University of Southampton Malaysia \\ 3, Persiaran Canselor 1, Kota Ilmu Educity@ Iskandar, 79200 Iskandar Puteri, Johor, \\ Malaysia
}

\begin{abstract}
Two forms of dynamic transfer matrix are derived for a one-dimensional (beam-like) repetitive pin-jointed structure with point masses located at nodal cross-sections, the displacement-force transfer matrix $\mathbf{G}$, and the displacement-displacement transfer matrix, $\mathbf{H}$. Similarity matrices are introduced to relate $\mathbf{G}$ and $\mathbf{H}$, together with their respective metrics. Symplectic orthogonality relationships for the eigenvectors of both $\mathbf{G}$ and $\mathbf{H}$ are derived, together with relationships between their respective sets of eigenvectors. New expressions for the group velocity are derived. For repetitive structures of finite length, natural frequency equations are derived employing both $\mathbf{G}$ and $\mathbf{H}$, including phase-closure and the direct application of boundary (end) conditions. Besides an exposition of the theory, some familiar but much new, the focus of the present paper is on the relationships between the two forms of transfer matrix, including their respective (dis)advantages. Numerical results, together with further theory necessary for interpretation, are presented in companion papers.
\end{abstract}

Key words: wave propagation; repetitive structures; transfer matrices; symplectic orthogonality; group velocity

* corresponding author

E-mail addresses: aka104@soton.ac.uk (A.K. Ashari)

ngs@soton.ac.uk (N.G. Stephen) 


\section{Nomenclature}

$A$

cross-sectional area

$c_{\mathrm{g}} \quad$ group velocity

d, $\mathbf{D}, D \quad$ displacement vectors, components

E $\quad$ Young's modulus of elasticity

f, $\mathbf{F}, F \quad$ force vectors, components

G displacement-force transfer matrix

H displacement-displacement transfer matrix

i, $i, \mathbf{I} \quad \sqrt{-1}$, index, identity matrix

$j, \mathbf{J} \quad$ index, metric matrix

$k, \mathbf{K} \quad$ bar stiffness, stiffness matrix

L, $L, \mathbf{L} \quad$ left, bar length, similarity matrix

$m, \mathbf{m} \quad$ mass, mass matrix

$n, N, \mathbf{N} \quad$ index, number of cells, similarity matrix

$\mathrm{R}, \mathbf{R} \quad$ right, reflection matrix

$\mathbf{S}$

generic state vector

$t, \mathbf{T} \quad$ time, generic transfer matrix

(right) eigenvector of $\mathbf{G}$, matrix of eigenvectors

$\mathbf{w}, \mathbf{W}^{\mathrm{T}} \quad$ wave amplitude vector, left eigenvector of $\mathbf{G}$

$\mathbf{X}$

(right) eigenvector of $\mathbf{H}$, matrix of eigenvectors

$\mathbf{Y}^{\mathrm{T}} \quad$ left eigenvector of $\mathbf{H}$

$\delta \quad$ decay rate constant

$\varphi$

phase change constant, or wavenumber

$\lambda, \Lambda \quad$ eigenvalue, matrix of eigenvalues 
$\omega, \boldsymbol{\Omega} \quad$ radian frequency, skew-symmetric matrix

\section{Introduction}

Repetitive or periodic structures consist of a cell which spatially repeats in one-, two- or three-dimensions. Each cell is connected to another in a regular pattern to form the complete structure. Such constructions are widely employed in engineering, and include rail track, turbine blade assemblies (bladed discs), building frameworks, cranes, aircraft fuselages, trusses and honeycomb panels. Since the manufacture and construction of such structures can also be a repetitive process, they represent a cost effective design solution for many engineering applications. Early contributions are described in references [1-7]. The joints between the structural members can be designed so that they allow additional degrees of freedom, providing the possibility of a change in structural shape [8], or to become a deployable mechanism/structure [9]. Furthermore, repetitive structures portray symmetrical features and often have an aesthetically pleasing appearance.

The present work is concerned with one-dimensional (beam-like) repetitive structures. When periodicity is taken into account, the static and dynamic analysis of an entire structure can be reduced to the analysis of a single repeating cell, together with boundary (end) conditions if the structure is not of infinite extent; equivalent continuum properties can be determined for segmented structures such as trusses [10]. The primary approach is through the use of a transfer matrix $\mathbf{T}$, which relates state vector components on the right-hand side to those on the left-hand side of the cell, i.e. $\mathbf{s}_{\mathrm{R}}=\mathbf{T} \mathbf{s}_{\mathrm{L}}$. (Alternative analytical approaches, including the receptance method, are described in Mead's 1996 review paper [7].) An eigenvector of the transfer matrix describes a pattern of state vector components which is unique to within a scalar multiplier, $\lambda$. Translational symmetry demands that this pattern is preserved as one moves from the left-hand to the right-hand side of the cell, allowing one to write $\mathbf{s}_{\mathrm{R}}=\lambda \mathbf{s}_{\mathrm{L}}$; this immediately leads to the standard eigenvalue problem $\mathbf{T} \mathbf{s}_{\mathrm{L}}=\lambda \mathbf{s}_{\mathrm{L}}$, or $(\mathbf{T}-\lambda \mathbf{I}) \mathbf{s}_{\mathrm{L}}=\mathbf{0}$. There are two forms of the transfer matrix $\mathbf{T}$ in frequent use: the first and more common [3,10-18,23-25] relates a state vector $\mathbf{S}$ of displacement and force components on either side of the cell, here presented as transfer matrix $\mathbf{G}$; the second and less common form [8,19-22] relates state vectors $\mathbf{S}$ of displacements at three consecutive nodal cross-sections of the complete structure, here presented as $\mathbf{H}$. 
Both $\mathbf{G}$ and $\mathbf{H}$ can be determined from the stiffness matrix $\mathbf{K}$; the latter is symmetric for linear elastic displacements and, in turn, both transfer matrices are symplectic, that is they satisfy a relationship of the form $\mathbf{T}^{\mathrm{T}} \mathbf{\Omega} \mathbf{T}=\boldsymbol{\Omega}$, where $\mathbf{T}^{\mathrm{T}}$ denotes the transpose of $\mathbf{T}$, and $\boldsymbol{\Omega}$ is a skew-symmetric matrix, known as the metric. For transfer matrix $\mathbf{G}$, the metric takes its simplest, canonical, form, and is written as $\mathbf{J}=\left[\begin{array}{cc}\mathbf{0} & \mathbf{I} \\ -\mathbf{I} & \mathbf{0}\end{array}\right]$. The significance of the metric is rooted in Hamiltonian mechanics. The metric in a Euclidean vector space is the length of a vector, calculated as the square root of the dot product of the vector with itself, an inner product. However, in a symplectic vector space, where the state vector consists of both displacement and force components, such an inner-product has no physical meaning. Instead, a symplectic inner product, employing $\mathbf{J}$ as the metric, multiplies displacement with force which is work or energy; rather than length, it is an area, which is preserved during (here spatial) evolution. Ultimately, it implies conservation of energy.

For the static problem, the force-displacement transfer matrix $\mathbf{G}$ is perhaps the more appropriate, as one can readily identify force resultants; thus the decay modes associated with self-equilibrated loading (as anticipated by Saint-Venant's principle), the rigid body modes associated with zero force components, and the transmission modes associated with the force resultants of tension, bending moment and shearing force can be easily recognised. This static problem is characterised by multiple unity eigenvalues for the rigid body and transmission modes. In turn the transfer matrix cannot be diagonalised, but can only be reduced to a Jordan canonical block form; e.g. the principal vector describing tension is coupled with the eigenvector for a rigid-body displacement in the axial $x$-direction within a $2 \times 2$ block [10].

For wave propagation, the displacement-displacement transfer matrix $\mathbf{H}$ is perhaps the more appropriate, as waves are naturally described in terms of their displacement characteristics, e.g. extensional, flexural, thickness-shear, rather than force resultants.

For the dynamic problem considered here, irrespective of whether one employs $\mathbf{G}$ or $\mathbf{H}$, repeating eigenvalues are unusual for a given frequency; it generally implies a crossing of branches on a dispersion diagram, when the eigenvectors are distinct. The exception is a socalled Krein collision [31], which occurs when one has equal eigenvalues at the same frequency, and one of the propagation modes displays anomalous dispersion, the other normal; this is considered in detail in a companion paper. 
Each formulation has advantages and disadvantages: for example, symplectic orthogonality of the eigenvectors of $\mathbf{G}$ is seen to be a special case of the Reciprocal Theorem of BettiMaxwell [26], whereas the weighted symplectic orthogonality of the eigenvectors of $\mathbf{H}$ is not so obviously related. On the other hand, a new expression for the group velocity $[15,16,27,28]$ is much more succinct when one employs $\mathbf{H}$. For a repetitive structure having finite length, boundary conditions are generally expressed in terms of zero force or zero displacement so transfer matrix $\mathbf{G}$ is the more appropriate. If the number of repeating cells $N$ is small, then the natural frequencies can be determined from a zero value determinant of a partition of $\mathbf{G}^{N}$; if one employs transfer matrix $\mathbf{H}$, then since the force vector does not naturally feature it has to be introduced at a free end, which leads to a frequency equation which involves $\mathbf{H}^{N-1}$. However, both of these methods become inaccurate when the number of cells is large, and natural frequencies are then most accurately found using phase-closure for $\mathbf{G}$.

This paper presents relationships between the two forms of transfer matrix, $\mathbf{G}$ and $\mathbf{H}$, between their respective eigenvectors and also orthogonality relationships. Some of the results are not new but re-derived in a concise formulation and provided here as a convenient resource for comparison with new results. The results are formulated for the dynamic problem, but apply equally for the static case, $\omega=0$.

The example structure consists of an (in)finite planar framework of pin-jointed members with a point mass $m$ located at each pin-joint, as shown in Figure 1(a). The indices $(n-1), n$ and $(n+1)$ denote three consecutive nodal cross-sections of the framework, and numbers 1, 2 and 3 denote tiers of the masses. Horizontal and vertical rods, assumed massless, have stiffness $k=E A / L$ where $E$ is the Young's modulus, $A$ is the cross-sectional area and $L$ is the rod's length. The cross-sectional area of the diagonal members, also assumed massless, is taken to be one-half of the horizontal and vertical members, so their stiffness is $k /(2 \sqrt{2})$. The diagonal members are not pinned where they cross. The structure is, in fact, identical to that which has been subject to extensive elastostatic eigenanalysis by Stephen and co-workers [10,23-25], but with the addition of point masses at the nodal cross-sections.

The complete structure can be regarded as a repetition of two possible forms of repeating entity, as shown in Figure 1(b) and 1(c). In Figure 1(b), the vertical members and the crosssectional masses are regarded as being shared between adjacent cells, therefore their stiffness 
and mass must be halved; for a pin-jointed rod, this is accomplished by taking the crosssectional area as $A / 2$. Similarly, the masses are drawn as semi-circles, to denote the fact that each has mass $m / 2$. This repeating cell is the more appropriate for the construction of transfer matrix G. On the other hand, the structure can be regarded as a repetition of nodal cross-sections, Figure 1(c), and this is the more appropriate for the construction of transfer matrix $\mathbf{H}$; the vertical members are no longer shared, so the full stiffness (cross-sectional area) is now employed.

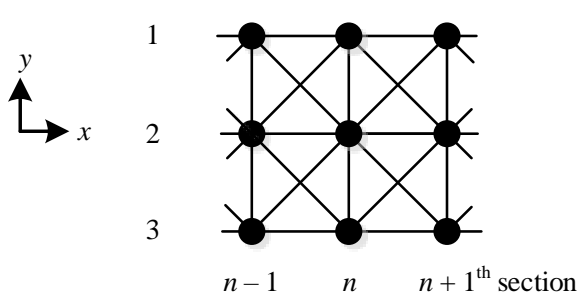

(a)

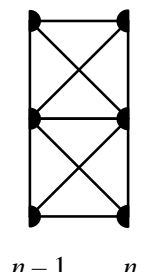

(L) $\quad(\mathrm{R})$

(b)

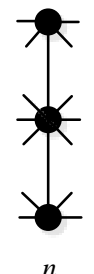

(c)

Figure 1. Segment of the example framework, (a), and two possible repeating entities; (b) shows the complete $n^{\text {th }}$ repeating cell, whereas (c) shows a repeating nodal cross-section with attached pin-jointed rods. For (b), the sections $(n-1)$ and $n$ can also be represented by left $(\mathrm{L})$ and right $(\mathrm{R})$, respectively.

\section{Formulation of the dynamic transfer matrices}

\subsection{Displacement-force transfer matrix, $\mathbf{G}$}

First consider the repeating cell shown in Figure 1(b); the governing equations of motion for the half-masses on sections $(n-1)$ and $(n)$ are

$$
\begin{gathered}
-\mathbf{f}(n-1)=\frac{\mathbf{m}}{2} \ddot{\mathbf{d}}(n-1)+\mathbf{K}_{\mathrm{LL}} \mathbf{d}(n-1)+\mathbf{K}_{\mathrm{LR}} \mathbf{d}(n), \\
\mathbf{f}(n)=\frac{\mathbf{m}}{2} \ddot{\mathbf{d}}(n)+\mathbf{K}_{\mathrm{RL}} \mathbf{d}(n-1)+\mathbf{K}_{\mathrm{RR}} \mathbf{d}(n),
\end{gathered}
$$

respectively, where $\mathbf{f}$ is the nodal force vector, $\mathbf{m}$ is the mass matrix, $\mathbf{d}$ is the displacement vector, and dot denotes differentiation with respect to time. The matrices $\mathbf{K}_{\mathrm{LL}}, \mathbf{K}_{\mathrm{LR}}, \mathbf{K}_{\mathrm{RL}}$ and $\mathbf{K}_{\mathrm{RR}}$ are presented explicitly in Appendix A. The mass matrix $\mathbf{m}$ is equal to $m$ times a 
$6 \times 6$ identity matrix. Following transfer matrix sign convention, the negative sign before $\mathbf{f}(n-1)$ is introduced to indicate that it acts in the opposite direction to $\mathbf{f}(n)$, that is, it acts in the negative $x$-direction. A factor of one-half for the mass matrix takes into account the halved point masses on both sides of the cell. For harmonic motion, one can write $\mathbf{f}(n)=\mathbf{F}(n) \exp (\mathrm{i} \omega t)$ and $\mathbf{d}(n)=\mathbf{D}(n) \exp (\mathrm{i} \omega t)$ where $\mathbf{F}$ and $\mathbf{D}$ are force and displacement amplitudes, respectively, $\mathrm{i}=\sqrt{-1}, \omega$ is frequency and $t$ is time. The acceleration vector can then be expressed as $\ddot{\mathbf{d}}(n)=-\omega^{2} \mathbf{D}(n) \exp (\mathrm{i} \omega t)$ which upon substitution into Eqs. (1) and (2) yields the dynamic stiffness matrix in partitioned form

$$
\left[\begin{array}{c}
-\mathbf{F}(n-1) \\
\mathbf{F}(n)
\end{array}\right]=\left[\begin{array}{cc}
{\left[\mathbf{K}_{\mathrm{LL}}-\frac{\omega^{2} \mathbf{m}}{2}\right]} & \mathbf{K}_{\mathrm{LR}} \\
& \mathbf{K}_{\mathrm{RL}}
\end{array}\right]\left[\begin{array}{c}
\mathbf{D}(n-1) \\
\left.\mathbf{R R}-\frac{\omega^{2} \mathbf{m}}{2}\right]
\end{array}\right] .
$$

Eq. (3) can be rearranged into transfer matrix form as

$$
\left[\begin{array}{l}
\mathbf{D}(n) \\
\mathbf{F}(n)
\end{array}\right]=\mathbf{G}\left[\begin{array}{l}
\mathbf{D}(n-1) \\
\mathbf{F}(n-1)
\end{array}\right]
$$

where

$$
\mathbf{G}=\left[\begin{array}{cc}
-\mathbf{K}_{\mathrm{LR}}^{-1}\left[\mathbf{K}_{\mathrm{LL}}-\frac{\omega^{2} \mathbf{m}}{2}\right] & -\mathbf{K}_{\mathrm{LR}}^{-1} \\
\mathbf{K}_{\mathrm{RL}}-\left[\mathbf{K}_{\mathrm{RR}}-\frac{\omega^{2} \mathbf{m}}{2}\right] \mathbf{K}_{\mathrm{LR}}^{-1}\left[\mathbf{K}_{\mathrm{LL}}-\frac{\omega^{2} \mathbf{m}}{2}\right] & \left.-\left[\mathbf{K}_{\mathrm{RR}}-\frac{\omega^{2} \mathbf{m}}{2}\right] \mathbf{K}_{\mathrm{LR}}^{-1}\right]
\end{array}\right]
$$

or more compactly $\mathbf{V}(n)=\mathbf{G V}(n-1)$, with $\mathbf{V}(n)=\left[\begin{array}{ll}\mathbf{D}^{\mathrm{T}}(n) & \mathbf{F}^{\mathrm{T}}(n)\end{array}\right]^{\mathrm{T}}$ and $\mathbf{V}(n-1)=\left[\begin{array}{ll}\mathbf{D}^{\mathrm{T}}(n-1) & \mathbf{F}^{\mathrm{T}}(n-1)\end{array}\right]^{\mathrm{T}}$.

The matrix $\mathbf{G}$ is symplectic, that is it satisfies the relationship

$$
\mathbf{G}^{\mathrm{T}} \mathbf{J G}=\mathbf{J}
$$

where $\mathbf{J}=\left[\begin{array}{cc}\mathbf{0} & \mathbf{I} \\ -\mathbf{I} & \mathbf{0}\end{array}\right]$ is the metric for $\mathbf{G}$ and has the properties $\mathbf{J}^{\mathrm{T}}=\mathbf{J}^{-1}=-\mathbf{J}$. The determinant of $\mathbf{G}$ is +1 for all values of $\omega$. The inverse of $\mathbf{G}$ is given by 
$\mathbf{G}^{-1}=\mathbf{J}^{-1} \mathbf{G}^{\mathrm{T}} \mathbf{J}=-\mathbf{J G}^{\mathrm{T}} \mathbf{J}$. Writing $\mathbf{V}(n)=\lambda \mathbf{V}(n-1)$, leads to the frequency-dependent eigenvalue problem

$$
\left[\begin{array}{cc}
-\mathbf{K}_{\mathrm{LR}}^{-1}\left[\mathbf{K}_{\mathrm{LL}}-\frac{\omega^{2} \mathbf{m}}{2}\right]-\lambda \mathbf{I} & -\mathbf{K}_{\mathrm{LR}}^{-1} \\
\mathbf{K}_{\mathrm{RL}}-\left[\mathbf{K}_{\mathrm{RR}}-\frac{\omega^{2} \mathbf{m}}{2}\right] \mathbf{K}_{\mathrm{LR}}^{-1}\left[\mathbf{K}_{\mathrm{LL}}-\frac{\omega^{2} \mathbf{m}}{2}\right] & \left.-\left[\mathbf{K}_{\mathrm{RR}}-\frac{\omega^{2} \mathbf{m}}{2}\right] \mathbf{K}_{\mathrm{LR}}^{-1}-\lambda \mathbf{I}\right]
\end{array}\right]\left[\begin{array}{l}
\mathbf{D}(n-1) \\
\mathbf{F}(n-1)
\end{array}\right]=\mathbf{0},
$$

or more compactly, $(\mathbf{G}-\lambda \mathbf{I}) \mathbf{V}(n-1)=0$.

\subsection{Displacement-displacement transfer matrix, $\mathbf{H}$}

Now consider the repeating nodal cross-section at an arbitrary station $n$, as shown in Figure 1(c). The governing equation of motion now takes the form

$$
\mathbf{m} \ddot{\mathbf{d}}(n)+\mathbf{K}_{\mathrm{RL}} \mathbf{d}(n-1)+\left[\mathbf{K}_{\mathrm{LL}}+\mathbf{K}_{\mathrm{RR}}\right] \mathbf{d}(n)+\mathbf{K}_{\mathrm{LR}} \mathbf{d}(n+1)=\mathbf{0} .
$$

(Referring to Appendix A, one sees that the term $k / 2$, pertaining to the one-half stiffness of the vertical members for Figure 1(b), only appears in the partitions $\mathbf{K}_{\mathrm{LL}}$ and $\mathbf{K}_{\mathrm{RR}}$; since these partitions are added in Eq. (7), this leads to the full stiffness of the vertical members in Figure 1(c).)

Writing $\mathbf{d}(n)=\mathbf{D}(n) \exp (\mathrm{i} \omega t)$ leads to

$$
\mathbf{K}_{\mathrm{RL}} \mathbf{D}(n-1)+\left[\mathbf{K}_{\mathrm{LL}}+\mathbf{K}_{\mathrm{RR}}-\omega^{2} \mathbf{m}\right] \mathbf{D}(n)+\mathbf{K}_{\mathrm{LR}} \mathbf{D}(n+1)=\mathbf{0} .
$$

Pre-multiplying by $\mathbf{K}_{\mathrm{LR}}^{-1}$ gives

$$
\mathbf{D}(n+1)=-\mathbf{K}_{\mathrm{LR}}^{-1} \mathbf{K}_{\mathrm{RL}} \mathbf{D}(n-1)-\mathbf{K}_{\mathrm{LR}}^{-1}\left[\mathbf{K}_{\mathrm{LL}}+\mathbf{K}_{\mathrm{RR}}-\omega^{2} \mathbf{m}\right] \mathbf{D}(n) .
$$

Introducing the state vectors

$$
\mathbf{X}(n-1)=\left[\begin{array}{ll}
\mathbf{D}^{\mathrm{T}}(n-1) & \mathbf{D}^{\mathrm{T}}(n)
\end{array}\right]^{\mathrm{T}} \text { and } \mathbf{X}(n)=\left[\begin{array}{ll}
\mathbf{D}^{\mathrm{T}}(n) & \mathbf{D}^{\mathrm{T}}(n+1)
\end{array}\right]^{\mathrm{T}}
$$

allows one to reconstruct Eq. (9) in the difference equation form

$$
\left[\begin{array}{c}
\mathbf{D}(n) \\
\mathbf{D}(n+1)
\end{array}\right]=\left[\begin{array}{cc}
\mathbf{0} & \mathbf{I} \\
-\mathbf{K}_{\mathrm{LR}}^{-1} \mathbf{K}_{\mathrm{RL}} & -\mathbf{K}_{\mathrm{LR}}^{-1}\left[\mathbf{K}_{\mathrm{LL}}+\mathbf{K}_{\mathrm{RR}}-\omega^{2} \mathbf{m}\right]
\end{array}\right]\left[\begin{array}{c}
\mathbf{D}(n-1) \\
\mathbf{D}(n)
\end{array}\right],
$$

or more compactly $\mathbf{X}(n)=\mathbf{H} \mathbf{X}(n-1)$. Finally, writing $\mathbf{X}(n)=\lambda \mathbf{X}(n-1)$ leads to the frequency-dependent eigenvalue problem 


$$
\left[\begin{array}{cc}
-\lambda \mathbf{I} & \mathbf{I} \\
-\mathbf{K}_{\mathrm{LR}}^{-1} \mathbf{K}_{\mathrm{RL}} & -\mathbf{K}_{\mathrm{LR}}^{-1}\left[\mathbf{K}_{\mathrm{LL}}+\mathbf{K}_{\mathrm{RR}}-\omega^{2} \mathbf{m}\right]-\lambda \mathbf{I}
\end{array}\right] \mathbf{X}(n-1)=\mathbf{0},
$$

or more compactly, $(\mathbf{H}-\lambda \mathbf{I}) \mathbf{X}(n-1)=0$. Note that matrix $\mathbf{H}$ is still symplectic, but does not satisfy Eq. (5), that is $\mathbf{H}^{\mathrm{T}} \mathbf{J H} \neq \mathbf{J}$; however, the equivalent relationship, together with a new metric, is developed in the following section.

\section{Relationships between transfer matrices $\mathbf{G}$ and $\mathbf{H}$}

The relationships between the dynamic transfer matrices $\mathbf{G}$ and $\mathbf{H}$ can be established as follows: let $\mathbf{L}$ and $\mathbf{N}$ be the matrices

$$
\mathbf{L}=\left[\begin{array}{cc}
\mathbf{I} & \mathbf{0} \\
-\left[\mathbf{K}_{\mathrm{LL}}-\frac{\omega^{2} \mathbf{m}}{2}\right] & -\mathbf{K}_{\mathrm{LR}}
\end{array}\right] \text { and } \mathbf{N}=\left[\begin{array}{cc}
\mathbf{0} & \mathbf{I} \\
\mathbf{K}_{\mathrm{RL}} & {\left[\mathbf{K}_{\mathrm{RR}}-\frac{\omega^{2} \mathbf{m}}{2}\right]}
\end{array}\right]
$$

then it is straightforward to show that

$$
\mathbf{G}=\mathbf{N} \mathbf{L}^{-1} \text { and } \mathbf{H}=\mathbf{L}^{-1} \mathbf{N} .
$$

The inverse of matrix $\mathbf{L}$ is found using the block-wise matrix inversion formula, Appendix B, as

$$
\mathbf{L}^{-1}=\left[\begin{array}{cc}
\mathbf{I} & \mathbf{0} \\
-\mathbf{K}_{\mathrm{LR}}^{-1}\left(\mathbf{K}_{\mathrm{LL}}-\omega^{2} \frac{\mathbf{m}}{2}\right) & -\mathbf{K}_{\mathrm{LR}}^{-1}
\end{array}\right] .
$$

Matrices $\mathbf{L}$ and $\mathbf{N}$ were first introduced by Zhong and Williams [19] as a means to avoid numerical ill-conditioning. Now, from Eqs. (14a,b) $\mathbf{G}^{\mathrm{T}}=\mathbf{L}^{-\mathrm{T}} \mathbf{N}^{\mathrm{T}}$ and $\mathbf{H}^{\mathrm{T}}=\mathbf{N}^{\mathrm{T}} \mathbf{L}^{-\mathrm{T}}$, so Eq. (5) becomes

$$
\left(\mathbf{L}^{-\mathrm{T}} \mathbf{N}^{\mathrm{T}}\right) \mathbf{J}\left(\mathbf{N L}^{-1}\right)=\mathbf{J}
$$

now pre-multiply by $\mathbf{N}^{\mathrm{T}}$ and post-multiply by $\mathbf{N}$ to give $\left(\mathbf{N}^{\mathrm{T}} \mathbf{L}^{-\mathrm{T}}\right) \mathbf{N}^{\mathrm{T}} \mathbf{J} \mathbf{N}\left(\mathbf{L}^{-1} \mathbf{N}\right)=\mathbf{N}^{\mathrm{T}} \mathbf{J} \mathbf{N}$ or $\mathbf{H}^{\mathrm{T}}\left(\mathbf{N}^{\mathrm{T}} \mathbf{J N}\right) \mathbf{H}=\mathbf{N}^{\mathrm{T}} \mathbf{J N}$. Write $\tilde{\mathbf{J}}=\mathbf{N}^{\mathrm{T}} \mathbf{J N}$, then we have $\mathbf{H}^{\mathrm{T}} \tilde{\mathbf{J}} \mathbf{H}=\tilde{\mathbf{J}}$ as the expression equivalent to Eq. (5) for matrix H. The metric is $\tilde{\mathbf{J}}=\left[\begin{array}{cc}\mathbf{0} & -\mathbf{K}_{\mathrm{LR}} \\ \mathbf{K}_{\mathrm{RL}} & \mathbf{0}\end{array}\right]$, and has the skewsymmetric property $\tilde{\mathbf{J}}^{\mathrm{T}}=-\tilde{\mathbf{J}}$ since $\mathbf{K}_{\mathrm{RL}}=\mathbf{K}_{\mathrm{LR}}^{\mathrm{T}} ;$ this allows one to write $\mathbf{H}^{-1}=\tilde{\mathbf{J}}^{-1} \mathbf{H}^{\mathrm{T}} \tilde{\mathbf{J}}$. The 
metric can also be expressed in terms of $\mathbf{L}$ : pre-multiply Eq. (15) by $\mathbf{L}^{\mathrm{T}}$ and post-multiply by $\mathbf{L}$ gives $\mathbf{N}^{\mathrm{T}} \mathbf{J N}=\mathbf{L}^{\mathrm{T}} \mathbf{J L}=\tilde{\mathbf{J}}$.

Matrices $\mathbf{G}$ and $\mathbf{H}$ are related as follows: from Eq. (14a), pre-multiply by $\mathbf{L}^{-1}$ to give $\mathbf{L}^{-1} \mathbf{G}=\left(\mathbf{L}^{-1} \mathbf{N}\right) \mathbf{L}^{-1}=\mathbf{H L}^{-1}$ and hence $\mathbf{G}=\mathbf{L} \mathbf{H L}^{-1}$, or equally $\mathbf{H}=\mathbf{L}^{-1} \mathbf{G} \mathbf{L}$. Pre-multiply Eq. (14b) by $\mathbf{N}$ to give $\mathbf{N H}=\left(\mathbf{N L}^{-1}\right) \mathbf{N}=\mathbf{G N}$ and hence $\mathbf{H}=\mathbf{N}^{-1} \mathbf{G N}$, or equally $\mathbf{G}=\mathbf{N H N}^{-1}$. Thus both $\mathbf{L}$ and $\mathbf{N}$ serve as similarity matrices for $\mathbf{G}$ and $\mathbf{H}$. Since $\mathbf{G}$ and $\mathbf{H}$ are similar, they must have the same eigenvalues, as one would expect, although the eigenvectors are quite different as will be noted below; however the eigenvectors are related, again through matrices $\mathbf{L}$ and $\mathbf{N}$. In the above, the inverse of $\mathbf{N}$ may be found by means of the Schur complement, Appendix B, as $\mathbf{N}^{-1}=\left[\begin{array}{cc}-\mathbf{K}_{\mathrm{RL}}^{-1}\left(\mathbf{K}_{\mathrm{RR}}-\omega^{2} \frac{\mathbf{m}}{2}\right) & \mathbf{K}_{\mathrm{RL}}^{-1} \\ \mathbf{I} & \mathbf{0}\end{array}\right]$. Similarly, $\tilde{\mathbf{J}}^{-1}=\left[\begin{array}{cc}\mathbf{0} & \mathbf{K}_{\mathrm{RL}}^{-1} \\ -\mathbf{K}_{\mathrm{LR}}^{-1} & \mathbf{0}\end{array}\right]$

\section{Eigenvalues and eigenvectors of transfer matrices $\mathbf{G}$ and $\mathbf{H}$}

The frequency-dependent eigenproblems, Eqs. $(6,12)$, can be solved computationally using the eig function of MATLAB. For a specified $\omega$, or $\omega^{2}$, MATLAB returns the eigenvalues $\lambda$ in a diagonal matrix and the corresponding normalised right eigenvectors $\mathbf{V}$ arranged accordingly in the eigenvector matrix, that is the eigenvector associated with the first eigenvalue will be in the first column of the eigenvector matrix, and so on.

For a cell with $2 N$ degrees of freedom, there are $N$ reciprocal pairs of eigenvalues: if the eigenvalue $\lambda_{i}$ is associated with a propagating or decaying wave from sections $(n-1)$ to $(n)$, that is from left-to-right, then its reciprocal pair $\lambda_{j}$ where $\lambda_{j}=1 / \lambda_{i}$ represents the same wave propagating or decaying from $(n)$ to $(n-1)$, that is from right-to-left. Usually, $\lambda$ is expressed in exponential form, i.e. $\lambda=\exp (\delta+\mathrm{i} \varphi)$ where $\delta$ and $\varphi$ represent decay rate and phase change of amplitudes from one section to the next, respectively. The eigenvalues take five possible forms [25]: 
(i) The positive real unity eigenvalue $\lambda=1=\exp (0)$ must occur an even number of times since its inverse will also be 1 .

(ii) The negative real unity eigenvalue $\lambda=-1=\exp (\mathrm{i} \pi)$ must also occur an even number of times since its inverse will be -1 .

(iii) The real non-unity eigenvalues occur as a pair $\lambda$ and $\lambda^{-1}$. If $\lambda>0$, then $\lambda=\exp (\delta)$, but if $\lambda<0$, then $\lambda=\exp (\delta+\mathrm{i} \pi)$.

(iv) The complex unity eigenvalues $|\lambda|=1$ occur as a unitary pair, that is $\lambda=a+\mathrm{i} b$ and $\lambda=a-\mathrm{i} b$. If $\lambda=\exp (\mathrm{i} \varphi)$, then $\lambda^{-1}=\exp (-\mathrm{i} \varphi)$; the inverse is also the complex conjugate.

(v) The complex non-unity eigenvalues occur as a quartet of reciprocals and complex conjugates, that is $\lambda=a+\mathrm{i} b, \lambda=a-\mathrm{i} b, \lambda=(a+\mathrm{i} b)^{-1}, \lambda=(a-\mathrm{i} b)^{-1}$; in exponential form, $\lambda=\exp ( \pm \delta \pm \mathrm{i} \varphi)$ where $\delta>0$ and $\varphi>0$. For example, if $\left|\lambda_{i}\right|<1$, then $\lambda_{i}=\exp (-\delta-\mathrm{i} \varphi)$, the complex conjugate is $\bar{\lambda}_{i}=\exp (-\delta+\mathrm{i} \varphi)$, the inverses are $\lambda_{i}^{-1}=\exp (+\delta+\mathrm{i} \varphi)$ and $\bar{\lambda}_{i}^{-1}=\exp (+\delta-\mathrm{i} \varphi)$. The minimum size of transfer matrix for complex non-unity eigenvalues to occur is $8 \times 8$, because the quartet of eigenvalues can multiply both a minimum four degrees of crosssectional displacement freedom, and four force components in the case of $\mathbf{G}$ or an additional four displacements in the case of $\mathbf{H}$; in turn the state-vector of both $\mathbf{G}$ or $\mathbf{H}$ will be $8 \times 1$.

For the model framework, the first six rows and the last six rows of the right eigenvector $\mathbf{V}$ of matrix $\mathbf{G}$ represent displacement and force components, respectively, at an arbitrary station $n-1$, i.e.

$$
\mathbf{V}^{\mathrm{T}}(n-1)=\left[\begin{array}{llllllllllll}
D_{1 x}^{n-1} & D_{1 y}^{n-1} & D_{2 x}^{n-1} & D_{2 y}^{n-1} & D_{3 x}^{n-1} & D_{3 y}^{n-1} & F_{1 x}^{n-1} & F_{1 y}^{n-1} & F_{2 x}^{n-1} & F_{2 y}^{n-1} & F_{3 x}^{n-1} & F_{3 y}^{n-1}
\end{array}\right]^{\mathrm{T}} .
$$

The first six rows and the last six rows of the right eigenvector $\mathbf{X}$ of matrix $\mathbf{H}$ represent displacement components at an arbitrary station $(n-1)$ and $(n)$, respectively, i.e.

$$
\mathbf{X}^{\mathrm{T}}(n-1)=\left[\begin{array}{llllllllllll}
D_{1 x}^{n-1} & D_{1 y}^{n-1} & D_{2 x}^{n-1} & D_{2 y}^{n-1} & D_{3 x}^{n-1} & D_{3 y}^{n-1} & D_{1 x}^{n} & D_{1 y}^{n} & D_{2 x}^{n} & D_{2 y}^{n} & D_{3 x}^{n} & D_{3 y}^{n}
\end{array}\right]^{\mathrm{T}} .
$$


These components of the eigenvectors associated with both transfer matrices are shown in Figure 2.

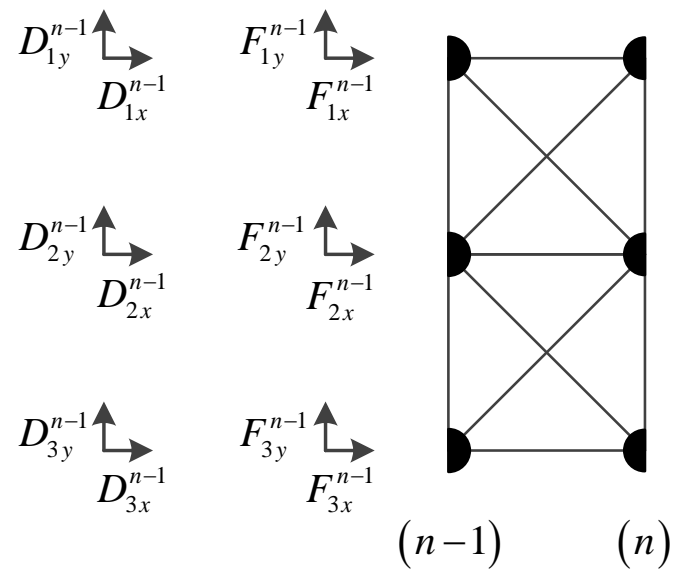

(a)

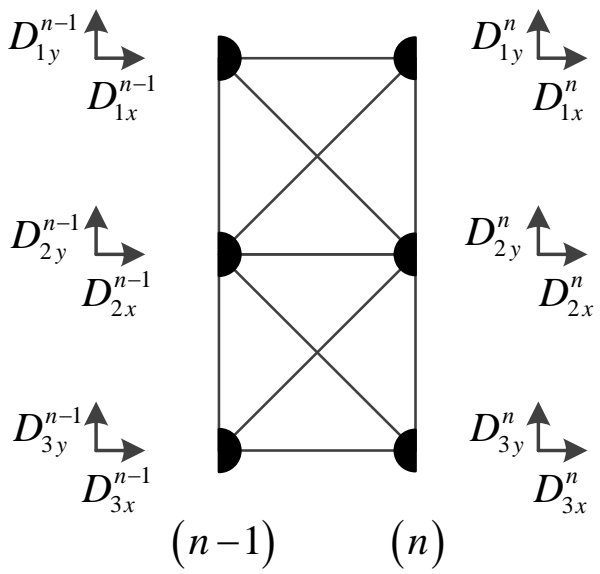

(b)

Figure 2. (a) Nodal displacements and forces pertaining to eigenvector $\mathbf{V}$ of transfer matrix G; (b) nodal displacements pertaining to eigenvector $\mathbf{X}$ of transfer matrix $\mathbf{H}$.

Despite the obvious difference between eigenvectors $\mathbf{V}$ and $\mathbf{X}$, they are related as follows: $\mathbf{V}$ is an eigenvector of $\mathbf{G}$, that is it satisfies $\mathbf{G V}=\lambda \mathbf{V} ; \mathbf{X}$ is an eigenvector of $\mathbf{H}$, that is it satisfies $\mathbf{H X}=\lambda \mathbf{X}$. But $\mathbf{G}=\mathbf{L H L} \mathbf{L}^{-1}$, or equally $\mathbf{H}=\mathbf{L}^{-1} \mathbf{G L}$; employing the former, one has $\left(\mathbf{L H} \mathbf{L}^{-1}\right) \mathbf{V}=\lambda \mathbf{V}$ and pre-multiplying by $\mathbf{L}^{-1}$ gives $\mathbf{H}\left(\mathbf{L}^{-1} \mathbf{V}\right)=\lambda\left(\mathbf{L}^{-1} \mathbf{V}\right)$ and hence $\mathbf{X}=\mathbf{L}^{-1} \mathbf{V}$, or $\mathbf{V}=\mathbf{L X}$.

But we also have $\mathbf{H}=\mathbf{N}^{-1} \mathbf{G N}$, or equally $\mathbf{G}=\mathbf{N H N ^ { - 1 }}$; employing the former, one has $\left(\mathbf{N}^{-1} \mathbf{G N}\right) \mathbf{X}=\lambda \mathbf{X}$ and pre-multiplying by $\mathbf{N}$ gives $\mathbf{G}(\mathbf{N X})=\lambda(\mathbf{N X})$ and hence $\mathbf{V}=\mathbf{N X}$. Employing the latter provides the equivalent $\mathbf{X}=\mathbf{N}^{-1} \mathbf{V}$, or $\mathbf{V}=\mathbf{N X}$.

From the above, it has been found that $\mathbf{V}=\mathbf{N X}$ and $\mathbf{V}=\mathbf{L X}$, that is both $\mathbf{L}$ and $\mathbf{N}$ serve equally to relate $\mathbf{X}$ to $\mathbf{V}$. However there is a subtlety that only becomes apparent when one expands these expressions: write $\mathbf{X}(n-1)=\left[\begin{array}{lll}\mathbf{D}^{\mathrm{T}}(n-1) & \mathbf{D}^{\mathrm{T}}(n)\end{array}\right]^{\mathrm{T}}$, then the former becomes 


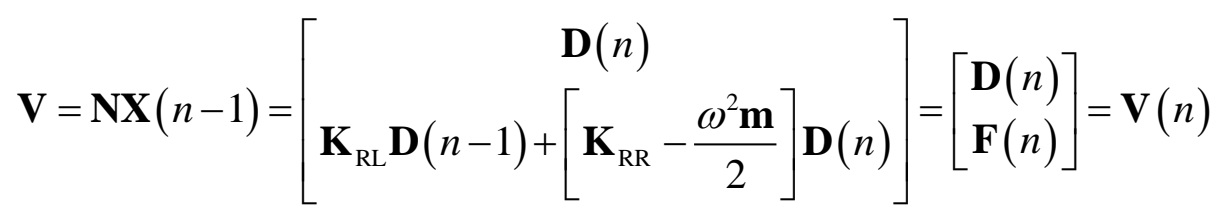

where the second row of Eq. (3) has been employed. Thus strictly, we have $\mathbf{V}(n)=\mathbf{N X}(n-1)$. For the latter,

$$
\mathbf{V}=\mathbf{L X}(n-1)=\left[\begin{array}{c}
\mathbf{D}(n-1) \\
-\left[\mathbf{K}_{\mathrm{LL}}-\frac{\omega^{2} \mathbf{m}}{2}\right] \mathbf{D}(n-1)-\mathbf{K}_{\mathrm{LR}} \mathbf{D}(n)
\end{array}\right]=\left[\begin{array}{l}
\mathbf{D}(n-1) \\
\mathbf{F}(n-1)
\end{array}\right]=\mathbf{V}(n-1)
$$

where the first row of Eq. (3) has been employed. Thus strictly, we have $\mathbf{V}(n-1)=\mathbf{L X}(n-1)$.

Finally, we derive expressions for the left eigenvectors of $\mathbf{G}$ and $\mathbf{H}$, as these are required in subsequent analysis to obtain the group velocity. The left eigenvector of matrix $\mathbf{G}$ can be evaluated as follows: let $\overline{\mathbf{V}}$ be the eigenvector associated with the eigenvalue $\lambda^{-1}$, i.e. $\mathbf{G} \overline{\mathbf{V}}=\lambda^{-1} \overline{\mathbf{V}}$ or $\mathbf{G}^{-1} \overline{\mathbf{V}}=\lambda \overline{\mathbf{V}}$; but $\mathbf{G}^{-1}=\mathbf{J}^{-1} \mathbf{G}^{\mathrm{T}} \mathbf{J}$, so we have $\mathbf{J}^{-1} \mathbf{G}^{\mathrm{T}} \mathbf{J} \overline{\mathbf{V}}=\lambda \overline{\mathbf{V}}$ or $\mathbf{G}^{\mathrm{T}}(\mathbf{J} \overline{\mathbf{V}})=\lambda(\mathbf{J} \overline{\mathbf{V}})$ which upon transposition gives $(\mathbf{J} \overline{\mathbf{V}})^{\mathrm{T}} \mathbf{G}=\lambda(\mathbf{J} \overline{\mathbf{V}})^{\mathrm{T}}$ or simply $\mathbf{W}^{\mathrm{T}} \mathbf{G}=\lambda \mathbf{W}^{\mathrm{T}}$ where $\mathbf{W}^{\mathrm{T}}=(\mathbf{J} \overline{\mathbf{V}})^{\mathrm{T}}$ is the left eigenvector of $\mathbf{G}$.

The left eigenvector of matrix $\mathbf{H}$ can be obtained by following a similar procedure: let $\overline{\mathbf{X}}$ be the eigenvector associated with the eigenvalue $\lambda^{-1}$, i.e. $\mathbf{H} \overline{\mathbf{X}}=\lambda^{-1} \overline{\mathbf{X}}$ or $\mathbf{H}^{-1} \overline{\mathbf{X}}=\lambda \overline{\mathbf{X}}$; but we know that $\mathbf{H}^{-1}=\tilde{\mathbf{J}}^{-1} \mathbf{H}^{\mathrm{T}} \tilde{\mathbf{J}}$, hence $\tilde{\mathbf{J}}^{-1} \mathbf{H}^{\mathrm{T}} \tilde{\mathbf{J}} \overline{\mathbf{X}}=\lambda \overline{\mathbf{X}}$ or $\mathbf{H}^{\mathrm{T}}(\tilde{\mathbf{J}} \overline{\mathbf{X}})=\lambda(\tilde{\mathbf{J}} \overline{\mathbf{X}})$ which upon transposition, gives $(\tilde{\mathbf{J}} \overline{\mathbf{X}})^{\mathrm{T}} \mathbf{H}=\lambda(\tilde{\mathbf{J}} \overline{\mathbf{X}})^{\mathrm{T}}$ or $\mathbf{Y}^{\mathrm{T}} \mathbf{H}=\lambda \mathbf{Y}^{\mathrm{T}}$ where $\mathbf{Y}^{\mathrm{T}}=(\tilde{\mathbf{J}} \overline{\mathbf{X}})^{\mathrm{T}}$ is the left eigenvector of $\mathbf{H}$.

\section{Symplectic orthogonality for transfer matrices $G$ and $H$}

Let $\mathbf{V}_{i}$ and $\mathbf{V}_{j}$ be eigenvectors of $\mathbf{G}$ associated with eigenvalues $\lambda_{i}$ and $\lambda_{j}$, respectively. Then one has $\mathbf{G} \mathbf{V}_{i}=\lambda_{i} \mathbf{V}_{i}$ and $\mathbf{G} \mathbf{V}_{j}=\lambda_{j} \mathbf{V}_{j}$. Transpose the former to give $\mathbf{V}_{i}^{\mathrm{T}} \mathbf{G}^{\mathrm{T}}=\lambda_{i} \mathbf{V}_{i}^{\mathrm{T}}$ and then post-multiply by $\mathbf{J G} \mathbf{V}_{j}$ to give $\mathbf{V}_{i}^{\mathrm{T}}\left(\mathbf{G}^{\mathrm{T}} \mathbf{J G}\right) \mathbf{V}_{j}=\lambda_{i} \mathbf{V}_{i}^{\mathrm{T}} \mathbf{J G} \mathbf{V}_{j}$ which reduces to 
$\mathbf{V}_{i}^{\mathrm{T}} \mathbf{J} \mathbf{V}_{j}=\lambda_{i} \lambda_{j} \mathbf{V}_{i}^{\mathrm{T}} \mathbf{J} \mathbf{V}_{j}$. Finally, rearrange as $\left(1-\lambda_{i} \lambda_{j}\right) \mathbf{V}_{i}^{\mathrm{T}} \mathbf{J} \mathbf{V}_{j}=0$ which implies that $\mathbf{V}_{i}^{\mathrm{T}} \mathbf{J} \mathbf{V}_{j}=0$ for $\lambda_{i} \neq 1 / \lambda_{j}$. This result shows that an eigenvector associated with an eigenvalue of the transfer matrix $\mathbf{G}$ is symplectic orthogonal to all other eigenvectors including itself with the exception of the eigenvector(s) associated with its reciprocal eigenvalue; the possible plurality of eigenvectors noted above can occur when one has a crossing of branches on a dispersion diagram, with a repeated eigenvalue and distinct eigenvectors. The symplectic orthogonality is a special case of the Betti-Maxwell reciprocal theorem as shown by Zhong and Williams [13,26] and later by Stephen [25]. For completeness, an implementation of the reciprocal theorem to obtain this expression is given in Appendix C. Zhong and Williams [13] have also shown that if $\mathbf{V}_{i}$ and $\mathbf{V}_{j}$ correspond to a reciprocal eigenpair, that is $\lambda_{i}=1 / \lambda_{j}$, then one can scale the eigenvector lengths such that $\mathbf{V}_{i}^{\mathrm{T}} \mathbf{J} \mathbf{V}_{j}=1$ and $\mathbf{V}_{j}^{\mathrm{T}} \mathbf{J} \mathbf{V}_{i}=-1$; however, as will be seen in a companion paper, this procedure loses information in the form of the Krein signature [31].

A similar procedure can be followed for matrix $\mathbf{H}$. Let $\mathbf{X}_{i}$ and $\mathbf{X}_{j}$ be the eigenvectors of $\mathbf{H}$ associated with eigenvalues $\lambda_{i}$ and $\lambda_{j}$, respectively. Then one has $\mathbf{H} \mathbf{X}_{i}=\lambda_{i} \mathbf{X}_{i}$ and $\mathbf{H} \mathbf{X}_{j}=\lambda_{j} \mathbf{X}_{j}$. Transpose the former to give $\mathbf{X}_{i}^{\mathrm{T}} \mathbf{H}^{\mathrm{T}}=\lambda_{i} \mathbf{X}_{i}^{\mathrm{T}}$ and then post-multiply by $\tilde{\mathbf{J}} \mathbf{H} \mathbf{X}_{j}$ to give $\mathbf{X}_{i}^{\mathrm{T}}\left(\mathbf{H}^{\mathrm{T}} \tilde{\mathbf{J}} \mathbf{H}\right) \mathbf{X}_{j}=\lambda_{i} \mathbf{X}_{i}^{\mathrm{T}} \tilde{\mathbf{J}} \mathbf{H} \mathbf{X}_{j}$ which reduces to $\mathbf{X}_{i}^{\mathrm{T}} \tilde{\mathbf{J}} \mathbf{X}_{j}=\lambda_{i} \lambda_{j} \mathbf{X}_{i}^{\mathrm{T}} \tilde{\mathbf{J}} \mathbf{X}_{j}$. Finally, rearrange as $\left(1-\lambda_{i} \lambda_{j}\right) \mathbf{X}_{i}^{\mathrm{T}} \tilde{\mathbf{J}} \mathbf{X}_{j}=0$ which implies that $\mathbf{X}_{i}^{\mathrm{T}} \tilde{\mathbf{J}} \mathbf{X}_{j}=0$ for $\lambda_{i} \neq 1 / \lambda_{j} ;$ again, for $\mathbf{X}_{i}$ and $\mathbf{X}_{j}$ which corresponds to a reciprocal eigenpair, the eigenvector lengths can be scaled such that $\mathbf{X}_{i}^{\mathrm{T}} \tilde{\mathbf{J}} \mathbf{X}_{j}=1$ and $\mathbf{X}_{j}^{\mathrm{T}} \tilde{\mathbf{J}} \mathbf{X}_{i}=-1$. In terms of the metric $\mathbf{J}$, this can be expressed as $\left(1-\lambda_{i} \lambda_{j}\right)\left(\mathbf{N} \mathbf{X}_{i}\right)^{\mathrm{T}} \mathbf{J}\left(\mathbf{N} \mathbf{X}_{j}\right)=0$ or equivalently $\left(1-\lambda_{i} \lambda_{j}\right)\left(\mathbf{L} \mathbf{X}_{i}\right)^{\mathrm{T}} \mathbf{J}\left(\mathbf{L} \mathbf{X}_{j}\right)=0$. This is referred to as weighted symplectic orthogonality.

\section{Group Velocity}

The velocity at which energy flows through a dispersive structure or medium is known as the group velocity $[15,16,27,28]$ and is defined as the derivative of frequency with respect to 
wavenumber, i.e. $c_{\mathrm{g}}=\frac{\mathrm{d} \omega}{\mathrm{d} \varphi}$. Using the chain rule $c_{\mathrm{g}}=\frac{\mathrm{d} \omega}{\mathrm{d} \varphi}=\frac{\mathrm{d} \omega}{\mathrm{d} \lambda} \frac{\mathrm{d} \lambda}{\mathrm{d} \varphi}$, and since $\lambda=\exp (\mathrm{i} \varphi)$, then $\frac{\mathrm{d} \lambda}{\mathrm{d} \varphi}=\mathrm{i} \exp (\mathrm{i} \varphi)=\mathrm{i} \lambda$; and one finds $c_{\mathrm{g}}=\mathrm{i} \lambda \frac{\mathrm{d} \omega}{\mathrm{d} \lambda}$. We now write matrix $\mathbf{H}$ in the form

$$
\mathbf{H}=\left[\begin{array}{cc}
\mathbf{0} & \mathbf{I} \\
-\mathbf{K}_{\mathrm{LR}}^{-1} \mathbf{K}_{\mathrm{RL}} & -\mathbf{K}_{\mathrm{LR}}^{-1}\left[\mathbf{K}_{\mathrm{LL}}+\mathbf{K}_{\mathrm{RR}}\right]
\end{array}\right]+\omega^{2}\left[\begin{array}{cc}
\mathbf{0} & \mathbf{0} \\
\mathbf{0} & \mathbf{K}_{\mathrm{LR}}^{-1} \mathbf{m}
\end{array}\right]
$$

or more compactly, $\mathbf{H}=\mathbf{H}_{0}+\omega^{2} \mathbf{H}_{2}$. The eigenvalue problem $[\mathbf{H}-\lambda \mathbf{I}] \mathbf{X}(n-1)=\mathbf{0}$, where $\mathbf{X}(n-1)=\left[\begin{array}{ll}\mathbf{D}^{\mathrm{T}}(n-1) & \mathbf{D}^{\mathrm{T}}(n)\end{array}\right]^{\mathrm{T}}$, can then be written as $\left[\mathbf{H}_{0}+\omega^{2} \mathbf{H}_{2}-\lambda \mathbf{I}\right] \mathbf{X}(n-1)=\mathbf{0}$.

Differentiating with respect to $\lambda$ gives

$$
\left[2 \omega \frac{\mathrm{d} \omega}{\mathrm{d} \lambda} \mathbf{H}_{2}-\mathbf{I}\right] \mathbf{X}+\left[\mathbf{H}_{0}+\omega^{2} \mathbf{H}_{2}-\lambda \mathbf{I}\right] \frac{\mathrm{d} \mathbf{X}}{\mathrm{d} \lambda}=\mathbf{0}
$$

where the index $(n-1)$ has been omitted. Let $\mathbf{Y}^{\mathrm{T}}$ be the left-eigenvector of matrix $\mathbf{H}$, pertaining to the same eigenvalue $\lambda$, then $\mathbf{Y}$ satisfies $\left[\mathbf{H}_{0}+\omega^{2} \mathbf{H}_{2}-\lambda \mathbf{I}\right]^{\mathrm{T}} \mathbf{Y}=\mathbf{0}$ or by transposing $\mathbf{Y}^{\mathrm{T}}\left[\mathbf{H}_{0}+\omega^{2} \mathbf{H}_{2}-\lambda \mathbf{I}\right]=\mathbf{0}$. So if we pre-multiply Eq. (19) by $\mathbf{Y}^{\mathrm{T}}$, the second term will disappear to give $\mathbf{Y}^{\mathrm{T}}\left[2 \omega \frac{\mathrm{d} \omega}{\mathrm{d} \lambda} \mathbf{H}_{2}-\mathbf{I}\right] \mathbf{X}=0$ or $\frac{\mathrm{d} \omega}{\mathrm{d} \lambda}=\frac{\mathbf{Y}^{\mathrm{T}} \mathbf{X}}{2 \omega \mathbf{Y}^{\mathrm{T}} \mathbf{H}_{2} \mathbf{X}}$. The group velocity is then

$$
c_{\mathrm{g}}=\mathrm{i} \lambda \frac{\mathbf{Y}^{\mathrm{T}} \mathbf{X}}{2 \omega \mathbf{Y}^{\mathrm{T}} \mathbf{H}_{2} \mathbf{X}} .
$$

A similar approach can be adopted for the matrix $\mathbf{G}$, which may be decomposed as $\mathbf{G}=\mathbf{G}_{0}+\omega^{2} \mathbf{G}_{2}+\omega^{4} \mathbf{G}_{4}$ where

$\mathbf{G}_{0}=\left[\begin{array}{cc}-\mathbf{K}_{\mathrm{LR}}^{-1} \mathbf{K}_{\mathrm{LL}} & -\mathbf{K}_{\mathrm{LR}}^{-1} \\ \mathbf{K}_{\mathrm{RL}}-\mathbf{K}_{\mathrm{RR}} \mathbf{K}_{\mathrm{LR}}^{-1} \mathbf{K}_{\mathrm{LL}} & -\mathbf{K}_{\mathrm{RR}} \mathbf{K}_{\mathrm{LR}}^{-1}\end{array}\right], \mathbf{G}_{2}=\left[\begin{array}{cc}\frac{\mathbf{m}}{2} \mathbf{K}_{\mathrm{LR}}^{-1} & \mathbf{0} \\ \frac{\mathbf{m}}{2}\left[\mathbf{K}_{\mathrm{LR}}^{-1} \mathbf{K}_{\mathrm{LL}}+\mathbf{K}_{\mathrm{RR}} \mathbf{K}_{\mathrm{LR}}^{-1}\right] & \frac{\mathbf{m}}{2} \mathbf{K}_{\mathrm{LR}}^{-1}\end{array}\right]$ and $\mathbf{G}_{4}=\left[\begin{array}{cc}\mathbf{0} & \mathbf{0} \\ -\frac{\mathbf{m}^{2}}{4} \mathbf{K}_{\mathrm{LR}}^{-1} & \mathbf{0}\end{array}\right]$; proceeding as above for the eigenvalue problem $[\mathbf{G}-\lambda \mathbf{I}] \mathbf{V}=0$ one finds $\frac{\mathrm{d} \omega}{\mathrm{d} \lambda}=\frac{\mathbf{W}^{\mathrm{T}} \mathbf{V}}{2 \omega \mathbf{W}^{\mathrm{T}} \mathbf{G}_{2} \mathbf{V}+4 \omega^{3} \mathbf{W}^{\mathrm{T}} \mathbf{G}_{4} \mathbf{V}}$ and hence group velocity 


$$
c_{\mathrm{g}}=\mathrm{i} \lambda \frac{\mathbf{W}^{\mathrm{T}} \mathbf{V}}{2 \omega \mathbf{W}^{\mathrm{T}} \mathbf{G}_{2} \mathbf{V}+4 \omega^{3} \mathbf{W}^{\mathrm{T}} \mathbf{G}_{4} \mathbf{V}},
$$

where $\mathbf{W}^{\mathrm{T}}$ is the left-eigenvector of matrix $\mathbf{G}$. For both Eqs. (20) and (21), we note that $c_{\mathrm{g}}$ is real because the product of $\lambda$ and $\mathrm{d} \omega / \mathrm{d} \lambda$ is purely imaginary.

Clearly, the expression for group velocity involving transfer matrix $\mathbf{H}$ is the more succinct, and since the $(12 \times 12)$ matrix $\mathbf{H}_{2}$ contains three $(6 \times 6)$ zero partitions, the structure of expression (20) can be revealed, as follows: since $\mathbf{X}(n-1)=\left[\begin{array}{ll}\mathbf{D}^{\mathrm{T}}(n-1) & \mathbf{D}^{\mathrm{T}}(n)\end{array}\right]^{\mathrm{T}}$, the product in the denominator $\mathbf{H}_{2} \mathbf{X}=\left[\begin{array}{ll}\mathbf{0} & m \mathbf{K}_{\mathrm{LR}}^{-1} \mathbf{D}^{\mathrm{T}}(n)\end{array}\right]^{\mathrm{T}}$ is a column vector, while from section 4 one has $\mathbf{Y}^{\mathrm{T}}=(\tilde{\mathbf{J}} \overline{\mathbf{X}})^{\mathrm{T}}$, or written in full $\mathbf{Y}^{\mathrm{T}}=\left[-\overline{\mathbf{D}}^{\mathrm{T}}(n) \mathbf{K}_{\mathrm{LR}}^{\mathrm{T}} \quad \overline{\mathbf{D}}^{\mathrm{T}}(n-1) \mathbf{K}_{\mathrm{RL}}^{\mathrm{T}}\right]=\left[-\overline{\mathbf{D}}^{\mathrm{T}}(n) \mathbf{K}_{\mathrm{RL}} \quad \overline{\mathbf{D}}^{\mathrm{T}}(n-1) \mathbf{K}_{\mathrm{LR}}\right]$ since $\mathbf{K}_{\mathrm{RL}}=\mathbf{K}_{\mathrm{LR}}^{\mathrm{T}}$. In turn, the denominator reduces to the scalar $\mathbf{Y}^{\mathrm{T}} \mathbf{H}_{2} \mathbf{X}=m \overline{\mathbf{D}}^{\mathrm{T}}(n-1) \mathbf{D}(n)$. The numerator is the scalar $\mathbf{Y}^{\mathrm{T}} \mathbf{X}=\overline{\mathbf{D}}^{\mathrm{T}}(n-1) \mathbf{K}_{\mathrm{LR}} \mathbf{D}(n)-\overline{\mathbf{D}}^{\mathrm{T}}(n) \mathbf{K}_{\mathrm{RL}} \mathbf{D}(n-1)$. If we now express all of the displacement vectors in term of cross-section index $(n-1)$, that is $\mathbf{D}(n)=\lambda \mathbf{D}(n-1)$ and $\overline{\mathbf{D}}(n)=\lambda^{-1} \mathbf{D}(n-1)$, the expression for the group velocity becomes

$$
c_{\mathrm{g}}=\frac{\mathrm{i} \overline{\mathbf{D}}^{\mathrm{T}}\left(\lambda \mathbf{K}_{\mathrm{LR}}-\lambda^{-1} \mathbf{K}_{\mathrm{RL}}\right) \mathbf{D}}{2 m \omega \overline{\mathbf{D}}^{\mathrm{T}} \mathbf{D}},
$$

where the $(n-1)$ index has been omitted. While not as succinct as Eq. (20), this form is suggestive of possible opposing contributions which could lead to both positive and negative group velocity, this signifying normal and anomalous dispersion, respectively. For the example structure, such anomalous dispersion does indeed occur as will be seen in a companion paper where it is explored in detail.

\section{Natural frequencies of finite length structures}

Finite length repetitive structures inevitably have boundary conditions, typically of zero displacement (fixed) or zero force (free), and for this reason the formulation employing transfer matrix $\mathbf{G}$ seems the more appropriate. For example, for a structure having $N$ cells, at 
a fixed left-hand end one immediately has $\mathbf{V}(0)=\left[\begin{array}{lll}\mathbf{D}^{\mathrm{T}}(0) & \mathbf{F}^{\mathrm{T}}(0)\end{array}\right]^{\mathrm{T}}=\left[\begin{array}{ll}\mathbf{0} & \mathbf{F}^{\mathrm{T}}(0)\end{array}\right]^{\mathrm{T}}$, whilst for a free right-hand end one has $\mathbf{V}(N)=\left[\begin{array}{lll}\mathbf{D}^{\mathrm{T}}(N) & \mathbf{F}^{\mathrm{T}}(N)\end{array}\right]^{\mathrm{T}}=\left[\begin{array}{ll}\mathbf{D}^{\mathrm{T}}(N) & \mathbf{0}\end{array}\right]^{\mathrm{T}}$. It is also possible to have mixed end conditions, for example some but not all of the nodal masses at either end could be fixed, the remaining free; for such cases, one can always re-cast the state vector column so that the first six elements are zero and the following six are unknown, and re-order the rows of the transfer matrix $\mathbf{G}$ accordingly. We now present three approaches to derive the frequency equation.

\subsection{Phase closure}

Phase closure $[29,30]$ is based upon the idea that for a standing wave, the total phase change for a complete circumnavigation of the structure is an integer multiple of $2 \pi$. The state vector $\left[\begin{array}{lll}\mathbf{D}^{\mathrm{T}}(n) & \mathbf{F}^{\mathrm{T}}(n)\end{array}\right]^{\mathrm{T}}$ at any given cross-section $n$ can only consist of the eigenvectors; how much of each is defined by participation coefficients, also known as the wave amplitudes $\mathbf{w}(n)$, and since some waves are propagating while others are decaying, the amount of each will be different at each cross-section. Thus we may write $\left[\begin{array}{ll}\mathbf{D}^{\mathrm{T}}(n) & \mathbf{F}^{\mathrm{T}}(n)\end{array}\right]^{\mathrm{T}}=\mathbf{V w}(n)$ where $\mathbf{V}$ is now an ordered $12 \times 12$ matrix containing the twelve eigenvectors of $\mathbf{G}$; in partitioned form

$$
\left[\begin{array}{l}
\mathbf{D}(n) \\
\mathbf{F}(n)
\end{array}\right]=\left[\begin{array}{ll}
\mathbf{V}_{\mathrm{R}} & \mathbf{V}_{\mathrm{L}}
\end{array}\right]\left[\begin{array}{l}
\mathbf{w}_{\mathrm{R}}(n) \\
\mathbf{w}_{\mathrm{L}}(n)
\end{array}\right]=\left[\begin{array}{ll}
\mathbf{V}_{\mathrm{R}, \mathrm{D}} & \mathbf{V}_{\mathrm{L}, \mathrm{D}} \\
\mathbf{V}_{\mathrm{R}, \mathrm{F}} & \mathbf{V}_{\mathrm{L}, \mathrm{F}}
\end{array}\right]\left[\begin{array}{l}
\mathbf{w}_{\mathrm{R}}(n) \\
\mathbf{w}_{\mathrm{L}}(n)
\end{array}\right]
$$

where the subscripts $\mathrm{R}$ and $\mathrm{L}$ refer to right- and left-going waves, respectively, while subscripts D and F refer to the displacement and force vectors, respectively. The order of columns of $V_{R}$ and $V_{L}$ must correspond to their eigenvalue pairs.

Now introduce reflection matrices $\mathbf{R}$ which describe the effect of the end-condition on each of the impinging wave amplitudes. Consider a finite length structure of $N$ cells. For a free left-hand end, the force vector is $\mathbf{F}(0)=\mathbf{0}$; from the second row of Eq. (23) one has $\mathbf{V}_{\mathrm{R}, \mathrm{F}} \mathbf{w}_{\mathrm{R}}(0)+\mathbf{V}_{\mathrm{L}, \mathrm{F}} \mathbf{w}_{\mathrm{L}}(0)=\mathbf{0}$, which may be arranged in the form $\mathbf{w}_{\mathrm{R}}(0)=\mathbf{R}(0) \mathbf{w}_{\mathrm{L}}(0)$, where $\mathbf{R}(0)=-\mathbf{V}_{\mathrm{R}, \mathrm{F}}^{-1} \mathbf{V}_{\mathrm{L}, \mathrm{F}}$ is the reflection matrix at the free left-hand end. Similarly, for a free right-hand end, the force vector is $\mathbf{F}(N)=\mathbf{0}$. Following the same procedure gives 
$\mathbf{w}_{\mathrm{L}}(N)=\mathbf{R}(N) \mathbf{w}_{\mathrm{R}}(N)$, where $\mathbf{R}(N)=-\mathbf{V}_{\mathrm{L}, \mathrm{F}}^{-1} \mathbf{V}_{\mathrm{R}, \mathrm{F}}$ is the reflection matrix at the free righthand end. Note that $\mathbf{R}(N)=\mathbf{R}^{-1}(0)$, which can be attributed to symmetry.

To apply the phase closure principle, consider right-going waves impinging upon the righthand end, station $N, \mathbf{w}_{\mathrm{R}}(N)$ which can be written in terms of $\mathbf{w}_{\mathrm{R}}(0)$ as $\mathbf{w}_{\mathrm{R}}(N)=\boldsymbol{\Lambda}_{\mathrm{R}}^{N} \mathbf{w}_{\mathrm{R}}(0)$ where $\boldsymbol{\Lambda}_{\mathrm{R}}$ is a diagonal eigenvalue matrix associated with right-going waves. By repeated substitution,

$$
\begin{aligned}
\mathbf{w}_{\mathrm{R}}(N) & =\boldsymbol{\Lambda}_{\mathrm{R}}^{N} \mathbf{w}_{\mathrm{R}}(0) \\
& =\boldsymbol{\Lambda}_{\mathrm{R}}^{N} \mathbf{R}(0) \mathbf{w}_{\mathrm{L}}(0) \\
& =\boldsymbol{\Lambda}_{\mathrm{R}}^{N} \mathbf{R}(0) \boldsymbol{\Lambda}_{\mathrm{R}}^{N} \mathbf{w}_{\mathrm{L}}(N) \\
& =\boldsymbol{\Lambda}_{\mathrm{R}}^{N} \mathbf{R}(0) \boldsymbol{\Lambda}_{\mathrm{R}}^{N} \mathbf{R}(N) \mathbf{w}_{\mathrm{R}}(N),
\end{aligned}
$$

or $\left[\boldsymbol{\Lambda}_{\mathrm{R}}^{N} \mathbf{R}(0) \boldsymbol{\Lambda}_{\mathrm{R}}^{N} \mathbf{R}(N)-\mathbf{I}\right] \mathbf{w}_{\mathrm{R}}(N)=\mathbf{0}$. For a standing wave to occur, one must have

$$
\operatorname{det}\left[\boldsymbol{\Lambda}_{\mathrm{R}}^{N} \mathbf{R}(0) \boldsymbol{\Lambda}_{\mathrm{R}}^{N} \mathbf{R}(N)-\mathbf{I}\right]=0 .
$$

Note that the determinant is complex, and the natural frequencies are found when the real and imaginary parts vanish simultaneously.

The formulation, Eq. (25), is unchanged for structures with different end conditions; what do change are the reflection matrices. These are summarised as follows:

Free left-hand end $\quad \mathbf{w}_{\mathrm{R}}(0)=\mathbf{R}(0) \mathbf{w}_{\mathrm{L}}(0)$ where $\mathbf{R}(0)=-\mathbf{V}_{\mathrm{R}, \mathrm{F}}^{-1} \mathbf{V}_{\mathrm{L}, \mathrm{F}}$

Fixed left-hand end $\quad \mathbf{w}_{\mathrm{R}}(0)=\mathbf{R}(0) \mathbf{w}_{\mathrm{L}}(0)$ where $\mathbf{R}(0)=-\mathbf{V}_{\mathrm{R}, \mathrm{D}}^{-1} \mathbf{V}_{\mathrm{L}, \mathrm{D}}$

Free right-hand end $\quad \mathbf{w}_{\mathrm{L}}(N)=\mathbf{R}(N) \mathbf{w}_{\mathrm{R}}(N)$ where $\mathbf{R}(N)=-\mathbf{V}_{\mathrm{L}, \mathrm{F}}^{-1} \mathbf{V}_{\mathrm{R}, \mathrm{F}}$

Fixed right-hand end $\mathbf{w}_{\mathrm{L}}(N)=\mathbf{R}(N) \mathbf{w}_{\mathrm{R}}(N)$ where $\mathbf{R}(N)=-\mathbf{V}_{\mathrm{L}, \mathrm{D}}^{-1} \mathbf{V}_{\mathrm{R}, \mathrm{D}}$

Two comments should be made: first, the $\mathbf{w}_{\mathrm{R}}(N)$ on the left-hand side of Eq. (24) is the same as that on the right-hand side only by virtue of a spatial phase change of an integer multiple of $2 \pi$. For the fundamental natural frequency, this phase change will be $2 \pi$, for the second $4 \pi$, and so on. Second, for a free vibration, right-going waves can only propagate, $|\lambda|=1$, or decay, $|\lambda|<1$, as one moves from left to right along the structure; thus each of the eigenvalues within matrix $\boldsymbol{\Lambda}_{\mathrm{R}}$ has modulus equal to or less than unity, and powers of $\boldsymbol{\Lambda}_{\mathrm{R}}$ 
will in turn only lead to terms whose modulus is equal to or less than unity. Similarly, leftgoing waves after reflection from the right-hand end can only propagate or decay as one moves from right to left along the structure, so one must still employ those eigenvalues whose modulus is equal to or less than unity, that is $\boldsymbol{\Lambda}_{\mathrm{R}}$, which is why one has employed $\mathbf{w}_{\mathrm{L}}(0)=\Lambda_{\mathrm{R}}^{N} \mathbf{w}_{\mathrm{L}}(N)$ in the development of Eq. (24). The reciprocal eigenvalue matrix, $\boldsymbol{\Lambda}_{\mathrm{L}}$, which contains eigenvalues with modulus equal to or greater than unity, does not therefore feature in the formulation, and this exclusion ensures numerical stability however large the value of $N$. The Riccati transfer matrix method, previously employed for the static analysis of the present structure [24], also excludes eigenvalues greater than unity, again ensuring numerical stability.

\subsection{Direct application of boundary conditions for matrix $\mathbf{G}$}

Again for a finite length structure of $N$ cells, the state vector at station $N$ is related to that at station 0 by

$$
\left[\begin{array}{l}
\mathbf{D}(N) \\
\mathbf{F}(N)
\end{array}\right]=\mathbf{G}^{N}\left[\begin{array}{l}
\mathbf{D}(0) \\
\mathbf{F}(0)
\end{array}\right] ;
$$

powers of $\mathbf{G}$ are computed most efficiently by noting that $\mathbf{G}=\mathbf{V} \mathbf{\Lambda} \mathbf{V}^{-1}$, so that $\mathbf{G}^{N}=\mathbf{V} \boldsymbol{\Lambda}^{N} \mathbf{V}^{-1}$. However, since the diagonal $\boldsymbol{\Lambda}$ contains both $\boldsymbol{\Lambda}_{\mathrm{L}}$ and $\boldsymbol{\Lambda}_{\mathrm{R}}$, powers of the former will become large for large $N$, leading to numerical instability. Introduce the notation $\mathbf{G}^{N}=\hat{\mathbf{G}}=\left[\begin{array}{ll}\hat{\mathbf{G}}_{\mathrm{DD}} & \hat{\mathbf{G}}_{\mathrm{DF}} \\ \hat{\mathbf{G}}_{\mathrm{FD}} & \hat{\mathbf{G}}_{\mathrm{FF}}\end{array}\right]$.

For free-free ends, $\mathbf{F}(0)=\mathbf{F}(N)=\mathbf{0}$, and from the second row of Eq. (26) one has $\hat{\mathbf{G}}_{\mathrm{FD}} \mathbf{D}(0)=\mathbf{0}$ and hence the frequency equation $\operatorname{det}\left(\hat{\mathbf{G}}_{\mathrm{FD}}\right)=0$. For fixed-fixed ends, $\mathbf{D}(0)=\mathbf{D}(N)=\mathbf{0}$ and from the first row of Eq. (26), one has $\hat{\mathbf{G}}_{\mathrm{DF}} \mathbf{F}(0)=\mathbf{0}$ and hence the frequency equation $\operatorname{det}\left(\hat{\mathbf{G}}_{\mathrm{DF}}\right)=0$. Finally, for fixed-free and free-fixed ends, the frequency equations are $\operatorname{det}\left(\hat{\mathbf{G}}_{\mathrm{FF}}\right)=0$ and $\operatorname{det}\left(\hat{\mathbf{G}}_{\mathrm{DD}}\right)=0$, respectively. Unlike phase-closure, these determinantal frequency equations employing transfer matrix $\mathbf{G}$ are real. 


\subsection{Direct application of boundary conditions for matrix $\mathbf{H}$}

Consider, again, a free-free structure of $N$ cells. Since the force vector does not naturally feature within this formulation, it has to be introduced at either end. For a free vibration, the equations of motion at the left- and right-hand ends are, respectively

$-\mathbf{F}(0)=\left[\mathbf{K}_{\mathrm{LL}}-\frac{\omega^{2} \mathbf{m}}{2}\right] \mathbf{D}(0)+\mathbf{K}_{\mathrm{LR}} \mathbf{D}(1)$, and $\mathbf{F}(N)=\mathbf{K}_{\mathrm{RL}} \mathbf{D}(N-1)+\left[\mathbf{K}_{\mathrm{RR}}-\frac{\omega^{2} \mathbf{m}}{2}\right] \mathbf{D}(N)$.

From the boundary condition $\mathbf{F}(0)=\mathbf{0}$ one has $\mathbf{D}(1)=-\mathbf{K}_{\mathrm{LR}}^{-1}\left[\mathbf{K}_{\mathrm{LL}}-\frac{\omega^{2} \mathbf{m}}{2}\right] \mathbf{D}(0)$, and hence for the first cell

$$
\mathbf{X}(0)=\left[\begin{array}{c}
\mathbf{D}(0) \\
\mathbf{D}(1)
\end{array}\right]=\left[\begin{array}{c}
\mathbf{I} \\
-\mathbf{K}_{\mathrm{LR}}^{-1}\left[\mathbf{K}_{\mathrm{LL}}-\frac{\omega^{2} \mathbf{m}}{2}\right]
\end{array}\right] \mathbf{D}(0)
$$

From the boundary condition $\mathbf{F}(N)=\mathbf{0}$ one has for the $N^{\text {th }}$ cell

$$
\left[\mathbf{K}_{\mathrm{RL}}\left[\mathbf{K}_{\mathrm{RR}}-\frac{\omega^{2} \mathbf{m}}{2}\right]\right] \mathbf{X}(N-1)=\mathbf{0},
$$

where $\mathbf{X}(N-1)=\left[\begin{array}{ll}\mathbf{D}^{\mathrm{T}}(N-1) & \mathbf{D}^{\mathrm{T}}(N)\end{array}\right]^{\mathrm{T}}$. Since

$$
\mathbf{X}(N-1)=\mathbf{H}^{N-1} \mathbf{X}(0)
$$

one immediately has

$$
\left[\mathbf{K}_{\mathrm{RL}}\left[\mathbf{K}_{\mathrm{RR}}-\frac{\omega^{2} \mathbf{m}}{2}\right]\right] \mathbf{H}^{N-1}\left[-\mathbf{K}_{\mathrm{LR}}^{-1}\left[\mathbf{K}_{\mathrm{LL}}-\frac{\omega^{2} \mathbf{m}}{2}\right]\right] \mathbf{D}(0)=\mathbf{0},
$$

and for a natural frequency

$$
\operatorname{det}\left(\left[\mathbf{K}_{\mathrm{RL}}\left[\mathbf{K}_{\mathrm{RR}}-\frac{\omega^{2} \mathbf{m}}{2}\right]\right] \mathbf{H}^{N-1}\left[-\mathbf{K}_{\mathrm{LR}}^{-1}\left[\mathbf{K}_{\mathrm{LL}}-\frac{\omega^{2} \mathbf{m}}{2}\right]\right]\right)=0,
$$

where $N \geq 1$ must be satisfied. 
For fixed-fixed ends, the left- and right-hand boundary conditions are $\mathbf{D}(0)=\mathbf{D}(N)=\mathbf{0}$, or in state vector form

$$
\begin{gathered}
\mathbf{X}(0)=\left[\begin{array}{l}
\mathbf{D}(0) \\
\mathbf{D}(1)
\end{array}\right]=\left[\begin{array}{l}
\mathbf{0} \\
\mathbf{I}
\end{array}\right] \mathbf{D}(1) \text { and } \\
{\left[\begin{array}{ll}
\mathbf{0} & \mathbf{I}
\end{array}\right] \mathbf{X}(N-1)=\mathbf{0},}
\end{gathered}
$$

where $\mathbf{X}(N-1)=\left[\begin{array}{ll}\mathbf{D}^{\mathrm{T}}(N-1) & \mathbf{D}^{\mathrm{T}}(N)\end{array}\right]^{\mathrm{T}}$, respectively. Substitute Eq. (32) into Eq. (29) and the resulting expression into Eq. (33) gives $\left[\begin{array}{ll}\mathbf{0} & \mathbf{I}\end{array}\right] \mathbf{H}^{N-1}\left[\begin{array}{ll}\mathbf{0} & \mathbf{I}\end{array}\right]^{\mathrm{T}} \mathbf{D}(1)=\mathbf{0}$, where $N>1$ must be satisfied (if $N=1$, then all the point masses are fixed, and vibration is impossible as the bars themselves are considered massless). For a natural frequency, one must have $\operatorname{det}\left(\left[\begin{array}{ll}\mathbf{0} & \mathbf{I}\end{array}\right] \mathbf{H}^{N-1}\left[\begin{array}{ll}\mathbf{0} & \mathbf{I}\end{array}\right]^{\mathrm{T}}\right)=0$. If we mimic the notation employed in the previous section, that is write $\mathbf{H}^{N-1}=\hat{\mathbf{H}}=\left[\begin{array}{ll}\hat{\mathbf{H}}_{11} & \hat{\mathbf{H}}_{12} \\ \hat{\mathbf{H}}_{21} & \hat{\mathbf{H}}_{22}\end{array}\right]$, then the determinantal frequency equation above reduces to the more succinct form $\operatorname{det}\left(\hat{\mathbf{H}}_{22}\right)=0$.

For fixed-free ends, the fixed left-hand end satisfies Eq. (32), while the free right-hand end satisfies Eq. (28); following the same procedures leads to

$$
\left[\mathbf{K}_{\mathrm{RL}}\left[\mathbf{K}_{\mathrm{RR}}-\frac{\omega^{2} \mathbf{m}}{2}\right]\right] \mathbf{H}^{N-1}\left[\begin{array}{l}
\mathbf{0} \\
\mathbf{I}
\end{array}\right] \mathbf{D}(1)=\mathbf{0},
$$

and for a natural frequency one has

$$
\operatorname{det}\left(\left[\mathbf{K}_{\mathrm{RL}}\left[\mathbf{K}_{\mathrm{RR}}-\frac{\omega^{2} \mathbf{m}}{2}\right]\right] \mathbf{H}^{N-1}\left[\begin{array}{l}
\mathbf{0} \\
\mathbf{I}
\end{array}\right]\right)=0 .
$$

For free-fixed ends, the free left-hand end satisfies Eq. (27) while the fixed right-hand end satisfies Eq. (33). Again, following the same procedures gives

$$
\left[\begin{array}{ll}
\mathbf{0} & \mathbf{I}
\end{array}\right] \mathbf{H}^{N-1}\left[-\mathbf{K}_{\mathrm{LR}}^{-1}\left[\mathbf{K}_{\mathrm{LL}}-\frac{\omega^{2} \mathbf{m}}{2}\right]\right] \mathbf{D}(0)=\mathbf{0},
$$

and for a natural frequency one has 


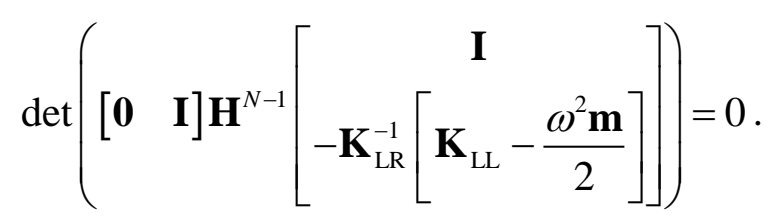

Again, these determinantal equations employing transfer matrix $\mathbf{H}$ are real.

As with $\mathbf{G}$, powers of $\mathbf{H}$ are computed most efficiently by noting that $\mathbf{H}=\mathbf{X} \mathbf{\Lambda} \mathbf{X}^{-1}$, so that $\mathbf{H}^{N-1}=\mathbf{X} \boldsymbol{\Lambda}^{N-1} \mathbf{X}^{-1}$, but again one can expect numerical instability as $N$ becomes large.

\section{Conclusions}

Two forms of dynamic transfer matrix have been derived for a one-dimensional (beam-like) repetitive pin-jointed structure with point masses located at nodal cross-sections, the displacement-force transfer matrix $\mathbf{G}$, and the displacement-displacement transfer matrix, $\mathbf{H}$. Wave propagation through the structure is described by the eigenvalues of either transfer matrix, expressed as $\lambda=\exp (\delta+\mathrm{i} \varphi)$, where the real part $\delta$ is the decay constant, and $\varphi$ is the phase constant as one moves from one cross-section to the next. The emphasis of the present paper has been the relationship between $\mathbf{G}$ and $\mathbf{H}$, and their respective properties, and also between their respective eigenvectors. Orthogonality relationships are derived for both sets of eigenvectors, allowing an arbitrary disturbance to be resolved into propagating and decaying waves. Both $\mathbf{G}$ and $\mathbf{H}$ can be employed to determine new expressions for the group velocity, but that which employs $\mathbf{H}$ is the more succinct.

For structures of finite length, the determination of natural frequencies of standing waves requires the application of boundary conditions, normally in terms of zero force (free-end), and/or zero displacement (fixed-end); accordingly, the displacement-force transfer matrix $\mathbf{G}$ is the more natural choice. Direct application of boundary conditions leads to very succinct frequency determinants based on partitions of $\mathbf{G}^{N}$. This approach is suitable for short beamlike structures, where the number of cells $N$ is small; however, since some of the eigenvalues are greater than unity, it becomes numerically unstable when $N$ is large. The displacementdisplacement transfer matrix $\mathbf{H}$ approach does not feature a force vector, so this needs to be introduced into the formulation, leading to less succinct frequency determinants; the exception is for the fixed-fixed beam. Again, this approach is numerically unstable when $N$ is large. Instead, phase-closure - the idea that for a standing wave, the total phase change for a complete circumnavigation of the structure is an integer multiple of $2 \pi$ - has been 
developed in terms of reflection matrices, and features only those eigenvalues less than or equal to unity; this ensures numerical stability whatever the magnitude of $N$. Both these approaches require the search for zero-values of a determinant; this can be done by evaluating and plotting the determinant over a frequency range, and noting where the value changes sign. For the direct method, the determinant is real, whereas for phase-closure, the determinant is complex, so both real and imaginary parts must be zero simultaneously.

Dispersion diagrams and numerical results for the example structure are considered in a companion paper.

\section{Appendix A}

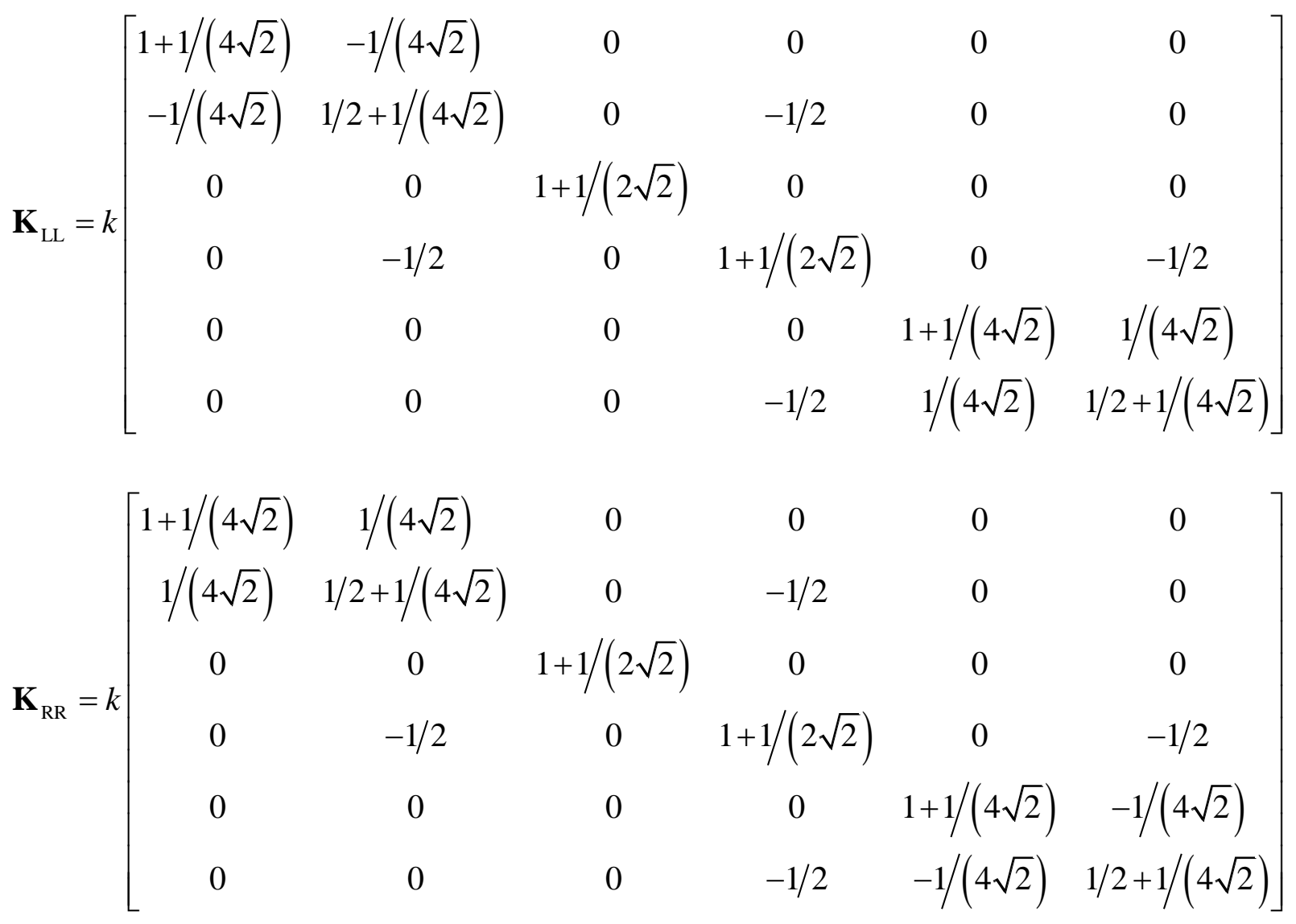




$$
\mathbf{K}_{\mathrm{LR}}=k\left[\begin{array}{cccccc}
-1 & 0 & -1 /(4 \sqrt{2}) & 1 /(4 \sqrt{2}) & 0 & 0 \\
0 & 0 & 1 /(4 \sqrt{2}) & -1 /(4 \sqrt{2}) & 0 & 0 \\
-1 /(4 \sqrt{2}) & -1 /(4 \sqrt{2}) & -1 & 0 & -1 /(4 \sqrt{2}) & 1 /(4 \sqrt{2}) \\
-1 /(4 \sqrt{2}) & -1 /(4 \sqrt{2}) & 0 & 0 & 1 /(4 \sqrt{2}) & -1 /(4 \sqrt{2}) \\
0 & 0 & -1 /(4 \sqrt{2}) & -1 /(4 \sqrt{2}) & -1 & 0 \\
0 & 0 & -1 /(4 \sqrt{2}) & -1 /(4 \sqrt{2}) & 0 & 0
\end{array}\right]
$$

where $k=E A / L$ and $\mathbf{K}_{\mathrm{RL}}=\mathbf{K}_{\mathrm{LR}}^{\mathrm{T}}$. Note that $\mathbf{K}_{\mathrm{LL}}$ and $\mathbf{K}_{\mathrm{RR}}$ are symmetric, but $\mathbf{K}_{\mathrm{LR}}$ is not.

\section{Appendix B}

If $\mathbf{A}, \mathbf{B}, \mathbf{C}$ and $\mathbf{D}$ are an $n \times n$, an $n \times m$, an $m \times n$ and an $m \times m$ matrix, respectively, then

$$
\left[\begin{array}{ll}
\mathbf{A} & \mathbf{B} \\
\mathbf{C} & \mathbf{D}
\end{array}\right]^{-1}=\left[\begin{array}{cc}
\left(\mathbf{A}-\mathbf{B D}^{-1} \mathbf{C}\right)^{-1} & -\mathbf{A}^{-1} \mathbf{B}\left(\mathbf{D}-\mathbf{C A}^{-1} \mathbf{B}\right)^{-1} \\
-\mathbf{D}^{-1} \mathbf{C}\left(\mathbf{A}-\mathbf{B D}^{-1} \mathbf{C}\right)^{-1} & \left(\mathbf{D}-\mathbf{C A}^{-1} \mathbf{B}\right)^{-1}
\end{array}\right]
$$

provided $|\mathbf{A}| \neq 0$ and $\left|\mathbf{D}-\mathbf{C A}^{-1} \mathbf{B}\right| \neq 0$, [32]. When $|\mathbf{A}|=0$, as with similarity matrix $\mathbf{N}$, one may use the Schur complement to give

$$
\left[\begin{array}{ll}
\mathbf{A} & \mathbf{B} \\
\mathbf{C} & \mathbf{D}
\end{array}\right]^{-1}=\left[\begin{array}{cc}
\left(\mathbf{A}-\mathbf{B D}^{-1} \mathbf{C}\right)^{-1} & -\left(\mathbf{A}-\mathbf{B D}^{-1} \mathbf{C}\right)^{-1} \mathbf{B D}^{-1} \\
-\mathbf{D}^{-1} \mathbf{C}\left(\mathbf{A}-\mathbf{B D}^{-1} \mathbf{C}\right)^{-1} & \mathbf{D}^{-1}+\mathbf{D}^{-1} \mathbf{C}\left(\mathbf{A}-\mathbf{B D}^{-1} \mathbf{C}\right)^{-1} \mathbf{B D}^{-1}
\end{array}\right]
$$

\section{Appendix C}

Consider a cell as shown in Figure 2(a). The cell is subjected to two different load systems denoted by subscripts 1 and 2. The Betti-Maxwell reciprocal theorem requires that the work done by forces $\mathbf{F}_{1}$ acting through displacements $\mathbf{D}_{2}$ is equal to the work done by forces $\mathbf{F}_{2}$ acting through displacements $\mathbf{D}_{1}$; however, we specialise load system 1 as the eigenvector $\mathbf{V}_{i}$ associated with eigenvalue $\lambda_{i}$, and load system 2 as the eigenvector $\mathbf{V}_{j}$ associated with eigenvalue $\lambda_{j}$, and the reciprocal theorem becomes 


$$
\mathbf{D}_{i}^{\mathrm{T}}(n-1) \mathbf{F}_{j}(n-1)+\mathbf{D}_{i}^{\mathrm{T}}(n) \mathbf{F}_{j}(n)=\mathbf{D}_{j}^{\mathrm{T}}(n-1) \mathbf{F}_{i}(n-1)+\mathbf{D}_{j}^{\mathrm{T}}(n) \mathbf{F}_{i}(n)
$$

where $(n-1)$ and $(n)$ are the LHS and RHS of the cell, respectively. Express the RHS vectors in terms of LHS vectors as follows

$$
\mathbf{D}_{i}(n)=\lambda_{i} \mathbf{D}_{i}(n-1), \mathbf{D}_{j}(n)=\lambda_{j} \mathbf{D}_{j}(n-1), \mathbf{F}_{i}(n)=-\lambda_{i} \mathbf{F}_{i}(n-1) \text { and } \mathbf{F}_{j}(n)=-\lambda_{j} \mathbf{F}_{j}(n-1)
$$

and the above simplifies to

$$
\left(1-\lambda_{i} \lambda_{j}\right) \mathbf{D}_{i}^{\mathrm{T}}(n-1) \mathbf{F}_{j}(n-1)=\left(1-\lambda_{i} \lambda_{j}\right) \mathbf{D}_{j}^{\mathrm{T}}(n-1) \mathbf{F}_{i}(n-1)
$$

or

$$
\left(1-\lambda_{i} \lambda_{j}\right)\left[\mathbf{D}_{i}^{\mathrm{T}}(n-1) \mathbf{F}_{j}(n-1)-\mathbf{D}_{j}^{\mathrm{T}}(n-1) \mathbf{F}_{i}(n-1)\right]=0 .
$$

But the term

$$
\left[\mathbf{D}_{i}^{\mathrm{T}}(n-1) \mathbf{F}_{j}(n-1)-\mathbf{D}_{j}^{\mathrm{T}}(n-1) \mathbf{F}_{i}(n-1)\right] \equiv \mathbf{V}_{i}^{\mathrm{T}} \mathbf{J} \mathbf{V}_{j},
$$

which indicates that the symplectic orthogonality relation $\left(1-\lambda_{i} \lambda_{j}\right) \mathbf{V}_{i}{ }^{\mathrm{T}} \mathbf{J} \mathbf{V}_{j}=0$ is a special case of the reciprocal theorem. Note that there is no equivalent direct link between the reciprocal theorem and the weighted symplectic orthogonality of the eigenvectors $\mathbf{X}$ of transfer matrix $\mathbf{H}$.

\section{References}

[1] F. Leckie, E. Pestel, Transfer-matrix fundamentals, International Journal of Mechanical Sciences 2(3) (1960) 137-167.

[2] F.W. Williams, Natural frequencies of repetitive structures, The Quarterly Journal of Mechanics and Applied Mathematics 24(3) (1971) 285-310.

[3] W.T. Thomson, Transfer matrix relationships for repeated structures, International Journal of Mechanical Sciences 13(8) (1971) 737-738.

[4] D.J. Mead, Wave propagation and natural modes in periodic systems: I. mono-coupled systems, Journal of Sound and Vibration 40(1) (1975) 1-18.

[5] D.J. Mead, Wave propagation and natural modes in periodic systems: II. multi-coupled systems, with and without damping, Journal of Sound and Vibration 40(1) (1975) 19-39. 
[6] R.C. Engels, Response of infinite periodic structures, Journal of Sound and Vibration 69(2) (1980) 181-197.

[7] D.J. Mead, Wave propagation in continuous periodic structures: research contributions from Southampton, 1964-1995, Journal of Sound and Vibration 190(3) (1996) 495-524.

[8] N. Wicks, S.D. Guest, Single member actuation in large repetitive truss structures, International Journal of Solids and Structures 41 (2004) 965-978.

[9] Z. You, S. Pellegrino, Foldable bar structures, International Journal of Solids and Structures 34 (1997) 1825-1847.

[10] N.G. Stephen, P.J. Wang, On Saint-Venant's principle in pin-jointed frameworks, International Journal of Solids and Structures 33 (1996) 79-97.

[11] Y. Yong, Y.K. Lin, Propagation of decaying waves in periodic and piecewise periodic structures of finite length, Journal of Sound and Vibration 129(2) (1989) 99-118.

[12] W.X. Zhong, F.W. Williams, On the localisation of the vibration mode of a substructural chain-type structure, Proceedings of the Institution of Mechanical Engineers, Part C Journal of Mechanical Engineering Science 205 (1991) 281-288.

[13] W.X. Zhong, F.W. Williams, Wave problems for repetitive structures and symplectic mathematics, Proceedings of the Institution of Mechanical Engineers, Part C Journal of Mechanical Engineering Science 206 (1992) 371-379.

[14] M. Zhou, W.X. Zhong, F.W. Williams, Wave propagation in structural chain-type structures excited by harmonic forces, International Journal of Mechanical Sciences 35 (1993) 953-964.

[15] R.S. Langley, A transfer matrix analysis of the energetics of structural wave motion and harmonic vibration, Proceedings of the Royal Society Series A 452 (1996) 1631-1648.

[16] J. Signorelli, A.H. von Flotow, Wave propagation, power flow, and resonance in a truss beam, Journal of Sound and Vibration 126(1) (1988) 127-144.

[17] B.R. Mace, D. Duhamel, M.J. Brennan, Finite element prediction of wave motion in structural waveguides, Journal of the Acoustical Society of America 117(5) (2005) 28352843. 
[18] D. Duhamel, B.R. Mace, M.J. Brennan, Finite element analysis of the vibrations of waveguides and periodic structures, Journal of Sound and Vibration 294 (2006) 205-220.

[19] W.X. Zhong, F.W. Williams, On the direct solution of wave propagation for repetitive structures, Journal of Sound and Vibration 181(3) (1995) 485-501.

[20] L. Gry, C. Gontier, Dynamic modelling of railway track: a periodic model based on a generalized beam formulation, Journal of Sound and Vibration 199(4) (1997) 531-558.

[21] Y. Waki, B.R. Mace, M.J. Brennan, Numerical issues concerning the wave and finite element method for free and forced vibrations of waveguides, Journal of Sound and Vibration 327 (2009) 92-108.

[22] E.G. Karpov, D.L. Dorofeev, N.G. Stephen, Characteristic solutions for the statics of repetitive beam-like trusses, International Journal of Mechanical Sciences 44(7) (2002) 1363-1379.

[23] N.G. Stephen, Repetitive beam-like structures: distributed loading and intermediate support, International Journal of Solids and Structures, 46(20) (2009) 3664-3668.

[24] N.G. Stephen, On the Riccati transfer matrix method for repetitive structures, Mechanics Research Communications 37(7) (2010) 663-665.

[25] N.G. Stephen, Transfer matrix analysis of the elastostatics of one-dimensional repetitive structures, Proceedings of the Royal Society Series A 462 (2006) 2245-2270.

[26] W.X. Zhong, F.W. Williams, Physical interpretation of the symplectic orthogonality of the eigensolutions of a Hamiltonian or symplectic matrix, Computers and Structures 49 (1993) 749-750.

[27] S. Finnveden, Evaluation of modal density and group velocity by a finite element method, Journal of Sound and Vibration 273 (2004) 51-75.

[28] M.N. Ichchou, S. Akrout, J.-M. Mencik, Guided waves group and energy velocities via finite elements, Journal of Sound and Vibration 305 (2007) 931-944.

[29] D.J. Mead, Waves and modes in finite beams: application of the phase-closure principle, Journal of Sound and Vibration 171(5) (1994) 695-702.

[30] H.M. Saeed, F. Vestroni, Simulation of combined systems by periodic structures: the wave transfer matrix approach, Journal of Sound and Vibration 213(1) (1998) 55-73.

[31] O.N. Kirillov, Nonconservative Stability Problems of Modern Physics, Studies in Mathematical Physics 14, De Gruyter, Berlin, 2013. 
[32] K. Ogata, Modern Control Engineering, Second Ed., Prentice Hall, USA, 1990. 